\title{
Gravidez Gemelar com Morte Fetal de Um dos Gêmeos: Avaliação Neurológica dos Gemelares Sobreviventes
}

\author{
Neurological Evaluation of the Surviving Twin When One Dies In Utero \\ Carolina Araújo Rodrigues Funayama ${ }^{1}$, Diva de Amorim Novaes², Fabrício da Silva Costa ${ }^{2}$, \\ Ricardo de Carvalho Cavalli², Geraldo Duarte ${ }^{2}$, \\ Sérgio Pereira da Cunha ${ }^{2}$
}

\begin{abstract}
RESUMO
Objetivo: verificar as intercorrências pré e perinatais e a evolução neurológica de crianças de gestações gemelares em que um dos fetos apresentou óbito intra-uterino.

Métodos: foram avaliados 14 casos de gestações gemelares ocorridas no periodo de 1988 a 1994 com óbito de um dos fetos, acompanhadas no Setor de Patologia Obstétrica do Hospital das Clinicas da Faculdade de Medicina de Ribeirão Preto da USP. Dados do acompanhamento pré e perinatal, bem como de autópsia dos fetos mortos, foram obtidos. As crianças foram convocadas no ano de 1996 para exame neurológico, sendo avaliados dados evolutivos e sinais patológicos nas áreas motoras, sensitivas, sensoriais e funções corticais superiores, incluindo linguagem, praxias e agnosias.

Resultados: do total de 14 casos, 10 retornaram para a avaliação neurológica tardia. Destas, apenas uma criança apresentou alteração no exame neurológico, cursando com paresia espástica de MIE de grau leve, tendo apresentado Apgar 0 no primeiro minuto e 5 no quinto minuto. A avaliação da placentação e idade gestacional mostrou 5 casos $(35,7 \%)$ com placentação monocoriônica e um caso com monoamniótica, 8 casos de pré-termo e 6 casos a termo. $O$ óbito de um dos fetos ocorreu no segundo trimestre em 6 casos $(42,8 \%)$ e os demais no terceiro trimestre.

Conclusões: o problema neurológico constatado em um único feto pode ser atribuído às intercorrências perinatais que este recém-nascido apresentou. Não tivemos como resultado outros RN com seqüelas provavelmente pela opção da conduta conservadora, tendo-se resolvido as gestações após a $32^{a}$ semana com diminuição das complicações do parto prétermo extremo.
\end{abstract}

PALAVRAS-CHAVE: Gemelaridade. Óbito fetal. Alterações neurológicas.

\section{Introdução}

A gemelaridade é condição sujeita a maior risco de morbidade neurológica perinatal em relação às gestações com feto único ${ }^{1}$. Possiviveis fatores de risco ou complicações da gravidez são mais freqüentes em gemelares, tais como idade avan-

${ }^{1}$ Departamento de Neurologia, Hospital das Clínicas da Faculdade de Medicina de Ribeirão Preto da USP.

2 Setor de Gestação de Alto Risco do Departamento de Ginecologia e Obstetrícia, Hospital das Clínicas da Faculdade de Medicina de Ribeirão Preto da USP.

Correspondência:

Ricardo de Carvalho Cavalli.

Rua Av. Caramuru, 2100, Apto. 1004 - Bairro República

14030-000 - Ribeirão Preto - SP çada, pré-eclâmpsia, anemia, poliidrâmnio, descolamento prematuro de placenta, corioamnionite, prolapso e circular de cordão ${ }^{1,2}$. Além do risco a que estão sujeitos devido a prematuridade e/ ou baixo peso ao nascimento, os gêmeos apresentam maior chance de ocorrência de hemorragia periventricular intra-uterina. Os gêmeos monozigóticos estão mais sujeitos a malformações, anormalidades cromossômicas, sindrome de transfusão feto-fetal e fenômenos tromboembólicos, como a coagulação intravascular disseminada, no caso de morte intra-uterina de um dos fetos ${ }^{3}$.

No periodo de 1988 a 1993, no Hospital das Clínicas de Ribeirão Preto - USP, observou-se taxa de $8 \%$ de morte intra-uterina em casos de 
gestação múltipla. Esta taxa é considerada superior à referida na literatura, que está entre 0,5 e $6,8 \%{ }^{4}$.

As gestações gemelares são mais suscetíveis ao nascimento pré-termo, restrição de crescimento intra-uterino e intercorrências neonatais. Estas intercorrências são causas importantes de alterações neurológicas neonatais, que também podem ser atribuídas a alterações hemodinâmicas após a morte de um dos fetos. Em gestações gemelares monocoriônicas com sindrome de transfusão feto-fetal caracterizadas por anastomose vascular placentária ocorrem hipoperfusão ao gêmeo sobrevivente e múltiplos infartos isquêmicos em orgãos do mesmo ${ }^{3}$.

A prematuridade extrema, nos casos de gestação menor que 32 semanas, apresenta altos indices de seqüelas neurológicas, com 13,4\% de paralisia cerebral e função cognitiva significativamente menor que no grupo controle ${ }^{5}$.

A avaliação ultra-sonográfica transfontanelar nos recém-nascidos provenientes de gemelares, com morte de um dos fetos logo após o nascimento, revelou lesões de maior e menor grau, caracterizando lesão cerebral adquirida no período antenatal ${ }^{6}$.

A perda de um dos fetos na gestação gemelar é relativamente freqüente, com risco maior na gemelaridade monocoriônica. Se a perda fetal ocorrer após a 16 semana de gestação, o gêmeo sobrevivente apresenta risco aumentado de morte intrauterina ${ }^{7}$. Em gemelaridade monocoriônica o risco de seqüela neurológica com morte unifetal pode chegar a $25 \%$, sendo que as lesões são conseqüência da hipoperfusão aguda imediatamente após a morte de um dos fetos ${ }^{7}$. O seguimento e a evolução destas gestações irão depender do tipo de placenta, da idade gestacional e das condições do gêmeo sobrevivente, lembrando-se que a prematuridade também é importante causa de morbimortalidade neonatal com possiveis seqüelas neurológicas ${ }^{5}$ o que pode dificultar a análise.

Para comparar as seqüelas observadas em crianças gêmeas, tanto aquelas decorrentes de condições perinatais associadas a prematuridade ou outras, como a do periodo de morte unifetal intrauterina, impõe-se investigação detalhada durante o período pré-natal e após o nascimento. O objetivo do presente trabalho foi verificar as intercorrências pré e perinatais e a evolução neurológica de crianças de gestações gemelares em que um dos fetos evoluiu para óbito intra-uterino.

\section{Pacientes e Métodos}

Foram analisados por meio de trabalho retrospectivo de coorte 14 casos de gestações geme- lares atendidos no Setor de Patologia Obstétrica do Hospital das Clinicas da Faculdade de Medicina de Ribeirão Preto (HCFMRP) no período de 1988 a 1994, em que um dos fetos foi a óbito intra-útero.

Dados de acompanhamento pré, peri e pósnatais, bem como de autópsia dos fetos mortos, foram obtidos dos protocolos de atendimento nos Serviços de Ginecologia e Obstetrícia e de Patologia do HCFMRP.

Os dados de acompanhamento pré e perinatais foram coletados pela análise de prontuário médico, com avaliação da idade materna em anos, paridade, idade gestacional do óbito unifetal e idade gestacional do parto, antecedentes pessoais e obstétricos, tipo de placenta e cavidade amniótica, tipo de parto e dados do recémnascido ao nascimento.

A idade gestacional em semanas foi definida pelo tempo de amenorréia em pacientes com ciclos menstruais regulares e com ultra-sonografia do primeiro trimestre compativel. Foi utilizada a idade gestacional obtida pela ultra-sonografia nos casos de data da última menstruação ignorada e/ ou ultra-sonografia do primeiro trimestre incompativel com o tempo de amenorréia.

Os dados do recém-nascido coletados ao nascimento foram peso, estatura e Apgar de primeiro minuto e quinto minuto.

As crianças sobreviventes foram convocadas, no ano de 1996, para exame clínico neurológico, realizado por neurologista e de acordo com o protocolo do Serviço de Neurologia Infantil deste hospital. Foram avaliados dados evolutivos e sinais patológicos nas áreas motoras, sensitivas, sensoriais e de funções corticais superiores, incluindo linguagem, praxias e agnosias.

O projeto foi submetido à avaliação da Comissão de Ética, com aprovação.

\section{Resultados}

Do total de 14 gestações gemelares e nascimentos acompanhados, 10 crianças retornaram ao serviço para a avaliação neurológica tardia. Houve 4 casos em que o seguimento foi perdido, tendo sido solicitado comparecimento por carta, sem resposta.

Em relação à idade materna, nove das 14 gestantes $(64,2 \%)$ tinham idade igual ou superior a 29 anos, com média de idade de 26,7 anos. Quanto a paridade, 6 pacientes eram primigestas e 8 pacientes tinham tido duas ou mais gestações. Cinco pacientes $(35,7 \%)$ possuiam doenças prévias à gravidez, sendo que uma delas tinha diagnóstico de alcoolismo e dependência a drogas ilicitas. Es- 
tes dados estão apresentados na Tabela 1 .

Em 5 (35,7\%) dos 14 casos, a placentação era monocoriônica, das quais uma era monoamniótica. O óbito de um dos fetos ocorreu no segundo trimestre em 6 casos (42\%) e em 8 casos (46\%) no terceiro trimestre. A idade gestacional da resolução variou de 24 a 41 semanas (Tabela 1).

Tabela 1 - Características de 14 casos de gravidez gemelar, com óbito unifetal intra-uterino.

\begin{tabular}{|c|c|c|c|c|c|}
\hline Caso & $\begin{array}{l}\text { Idade } \\
\text { (anos) }\end{array}$ & $\begin{array}{c}\text { Antecedentes } \\
\text { maternos }\end{array}$ & $\begin{array}{l}\text { Antecedentes } \\
\text { obstétricos }\end{array}$ & $\begin{array}{c}\text { Idade gestacional } \\
\text { (semanas) }\end{array}$ & Placentação \\
\hline 1 & 26 & $\mathrm{HAC}$ & G3P4AO & 41 & monoc/diam \\
\hline 2 & 30 & - & GIP2AO & 30 & dicor/diam \\
\hline 3 & 29 & $\mathrm{HAC}$ & GIP2AO & 28 & monoc/diam \\
\hline 5 & 38 & hipotiroidismo amaurose & GIP2AO & 40 & dicor/diam \\
\hline 6 & 24 & - & G3P3Al & 24 & dicor/diam \\
\hline 7 & 33 & - & G6P7A0 & 35 & monoc/diam \\
\hline 8 & 22 & - & GIP2AO & 37 & dicor/diam \\
\hline 9 & 33 & - & G3P4AO & 37 & dicor/diam \\
\hline 11 & 30 & Charcot-Marie & G3P2A2 & 35 & monoc/diam \\
\hline 12 & 29 & alcoolismo drogadição & G3P4AO & 37 & dicor/diam \\
\hline 13 & 27 & - & G3P5AO & 27 & tricor/triam \\
\hline 14 & 22 & - & GIP2AO & 38 & dicor/diam \\
\hline
\end{tabular}

Entre os natimortos detectou-se um caso de hidropsia não imune, em feto de mãe hipertensa crônica (caso 1) e outro com laudo anatomopatológico de hemorragia intraventricular (caso 7). Dois casos apresentavam malformações. O primeiro apresentava onfalocele, tetralogia de Fallot, hidroencefalia e persistência da veia cava superior esquerda (caso 11) e o outro, dilatação cística do quarto ventrículo (caso 13). Entre os nativivos não foram constatadas malformações.

Em relação às intercorrências neonatais, foi constatado um caso de enterocolite e hemorragia parenquimatosa intraventricular grau III (HPIV III) (caso 2), um caso com crises convulsivas e broncodisplasia (caso 3), um caso de membrana hialina, arritmia cardiaca, hipertensão pulmonar e HPIV III (caso 4) e um caso com fistula traqueo-esofágica com pertuito cervical (caso 14) (Tabela 2).

Em relação à idade gestacional, seis recémnascidos (RN) vivos eram a termo, entre os quais 4 pequenos para a idade gestacional (PIG), e os 8 casos restantes tiveram partos pré-termo, dos quais 5 eram PIG (Tabela 2).

Dos 14 recém-nascidos vivos (sobreviventes), apenas um apresentou indice de Apgar zero no primeiro minuto e 5 no $5^{\circ}$ minuto (Apgar $<7$ no $5^{\circ}$ min). Este RN teve várias intercorrências perinatais, caracterizadas por membrana hialina, hipertensão pulmonar e arritmia cardíaca, sendo o único que evoluiu com seqüela neurológica na avaliação tardia. Apresentou hemorragia intraventricular, constatada em exame de ultra-sonografia realizado uma semana após nascimento. No retorno tardio, encontrava-se com 5 anos e 8 meses e apresentava paresia espática de grau leve no membro inferior esquerdo.

\section{Discussão}

$\mathrm{Na}$ presente casuística ocorreu um número maior de gestantes com idade superior a 29 anos, o que está acima da média de nossa população, que é em torno de 22 anos. Este dado está de acordo com aquele observado no "National Center for Health Statistics" dos Estados Unidos no ano de $1990^{1}$.

$\mathrm{Na}$ avaliação das doenças maternas, observou-se que 3 gestantes (21\%) apresentavam hipertensão arterial crônica e nenhuma evoluiu para pré-eclâmpsia. Machado et al. ${ }^{2}$ relataram apenas uma caso com hipertensão arterial crônica (5,8\%) 
em 17 casos de gestação múltipla, e 80 (28\%) de 283 gestações únicas, sugerindo ser uma intercorrência pouco comum na gestação múltipla. Quanto à pré-eclâmpsia, entretanto, estes autores observaram uma taxa de $41,2 \%$ nas gestações múltiplas e 20,5\% nas gestações únicas, embora não tenham encontrado diferenças significativas nas freqüências e tipos de hipertensão arterial entre as duas populações estudadas.

Tabela 2 - Características dos recém-nascidos de 14 casos de gravidez gemelar, com óbito unifetal intra-uterino e de 10 examinadas em 1996

\begin{tabular}{|c|c|c|c|c|c|c|}
\hline \multirow{3}{*}{ Caso } & \multirow{3}{*}{$\begin{array}{c}\text { Natimorto } \\
\text { Peso (g) } \\
\text { óbito (trimestre) }\end{array}$} & \multicolumn{2}{|c|}{ Nativivo } & \multirow{3}{*}{$\begin{array}{l}\text { Intercorrências } \\
\text { neonatais }\end{array}$} & \multirow{2}{*}{\multicolumn{2}{|c|}{ Examinados em 1996}} \\
\hline & & \multirow[t]{2}{*}{ Peso (g) } & \multirow[t]{2}{*}{ Apgar (1/5 min) } & & & \\
\hline & & & & & Idade (anos/meses) & Avaliação neurológica \\
\hline 1 & 1.990 - macerado $\left(2^{\circ}\right)$ & $1.880-\mathrm{PIG}$ & $9 / 10$ & - & $5 a 7 m$ & normal \\
\hline 2 & 430 - macerado $\left(2^{\circ}\right)$ & 1.395 - PIG & $9 / 10$ & $\begin{array}{l}\text { enterocolite } \\
\text { HPIV-III }\end{array}$ & $4 a$ & normal \\
\hline 3 & 438 - macerado $\left(2^{\circ}\right)$ & $680-P I G$ & $3 / 10$ & $\begin{array}{l}\text { crises convulsivas } \\
\text { broncodisplasia }\end{array}$ & $3 a 5 m$ & normal \\
\hline 4 & $800\left(3^{\circ}\right)$ & $1.330-\mathrm{AIG}$ & $0 / 5$ & $\begin{array}{l}\text { HPIV-III-arritmia cardíaca } \\
\text { membrana hialina } \\
\text { hipert. pulmonar }\end{array}$ & $5 a 8 m$ & $\begin{array}{l}\text { paresia espática MIE } \\
\text { grau leve }\end{array}$ \\
\hline 5 & $2.640\left(3^{\circ}\right)$ & $3.670-$ AIG & $9 / 10$ & - & $2 a 3 m$ & normal \\
\hline 6 & macerado $\left(2^{\circ}\right)$ & 2.250 - PIG & $9 / 10$ & - & 3a $7 m$ & normal \\
\hline 7 & $1.640\left(3^{\circ}\right)$ & 2.260 - PIG & $7 / 10$ & desconforto respiratório & $8 m$ & normal \\
\hline 8 & $1.980\left(3^{\circ}\right)$ & 2.690 - PIG & $8 / 9$ & impetigo & - & \\
\hline 9 & 700 - mumificado $\left(2^{\circ}\right)$ & 2.330 - PIG & $8 / 9$ & icterícia fisiológica & - & \\
\hline 10 & macerado $\left(2^{\circ}\right)$ & $2.510-\mathrm{AIG}$ & $6 / 7$ & icterícia fisiológica & - & \\
\hline 11 & $2.170\left(3^{\circ}\right)$ & $1.860-\mathrm{AIG}$ & $9 / 10$ & - & $8 \mathrm{a} 11 \mathrm{~m}$ & normal \\
\hline 12 & $1.100\left(3^{\circ}\right)$ & $2.720-\mathrm{AIG}$ & $9 / 10$ & - & - & \\
\hline 13 & 2.310 e $900\left(3^{\circ}\right)$ & $1.430-\mathrm{PIG}$ & $7 / 9$ & - & $6 m$ & normal \\
\hline 14 & $1.300\left(3^{\circ}\right)$ & $2.300-P I G$ & $9 / 10$ & fístula tráqueo-esofágica & $2 a 6 m$ & normal \\
\hline
\end{tabular}

HIPV III: hemorragia parenquimatosa intraventricular GIII.

No presente estudo, dos 14 casos em que o feto foi a óbito, as causas não foram estabelecidas em 10 casos. Várias causas de morte fetal em gêmeos são citadas na literatura, entre elas casos isolados como trombose decorrente de enroscamento de cordão ${ }^{8}$, insuficiência vascular com comprometimento renal $^{9}$ e insuficiência placentária ${ }^{10}$.

Importância maior é dada à sindrome de transfusão feto-fetal na gestação gemelar monocoriônica, com aumento considerável da morbimortalidade perinatal. A morte de um dos fetos intra útero nos casos de gestação com sindrome de transfusão feto-fetal acarreta acréscimo nos riscos de lesão neurológica para o feto sobrevivente e risco de coagulação intravascular disseminada (CIVD) na gestante ${ }^{11,12}$. Com o óbito de um dos fetos, o gêmeo sobrevivente apresenta risco aumentado de fenômenos tromboembólicos graves ou complicações por hipoperfusão em vários órgãos, principalmente o cérebro. O dano ao cérebro, figa- do, baço e rim pode ser o resultado de transferência de proteínas pro-coagulantes do feto morto para o feto sobrevivente através da circulação placentária por anastomose vascular ou por aumento da pressão sanguínea intra-uterina após a morte de um dos fetos, com atividade uterina presente. Estudo recente postulou seqüestro agudo de sangue do feto sobrevivente sendo desviado para o feto morto através da anastomose da circulação placentária, levando a uma hipotensão aguda no feto sobrevivente, com dano cerebral irreversivel ${ }^{13}$.

Com a morte de um dos fetos no segundo ou terceiro trimestre gestacional, aumenta o risco de complicações e morte do feto sobrevivente, principalmente quando o quadro de coagulação intravascular disseminada (CIVD) se instala, sendo preponderante nos casos de óbito durante o terceiro trimestre ${ }^{14}$.

$\mathrm{Na}$ presente casuística houve 5 casos de placentação monocoriônica em que ocorreu mor- 
te unifetal no segundo ou terceiro trimestre, com apenas dois partos ocorridos a termo. Em um destes casos o RN sobrevivente apresentou seqüela neurológica, devido a eventos perinatais (hemorragia intraventricular), sendo realizada ultra-sonografia de crânio no final da primeira semana de vida, não demonstrando sinais antigos de lesão na substância branca ou cortical. Segundo Bejar et al. ${ }^{15,16}$, para ser considerada a possibilidade de necrose cerebral antenatal, o diagnóstico pela ultra-sonografia de necrose da substância branca deve ser feito até três dias após o nascimento.

É sabido que os riscos em uma gestação múltipla são bem maiores, seja ela monozigótica ou dizigótica. Entretanto, não podemos deixar de lembrar que estas gestações, geralmente, têm um parto mais precoce, de onde advém problema importante da prematuridade e suas seqüelas (baixo peso, membrana hialina, risco aumentado de infecções). Já é reconhecido que as seqüelas neurológicas estão também mais freqüentemente associadas às intercorrências neonatais, que são normalmente associadas com a prematuridade ${ }^{17,18}$

Em função destes dados, tem-se procurado protelar ao máximo a resolução gestacional precoce, mesmo nos casos de gestações múltiplas com óbito de um dos fetos, quando as avaliações mostram condições obstétricas favoráveis para a gestante e o feto sobrevivente ${ }^{19,20}$.

No presente trabalho, o distúrbio neurológico constatado em um único feto, no entanto, pode ser atribuído às intercorrências perinatais que este recém-nascido apresentou, em vez de ser considerado seqüela de óbito do outro gemelar. Não tivemos como resultado outros $\mathrm{RN}$ com seqüelas da prematuridade, provavelmente pela opção da conduta conservadora, tendo-se gestações com duração de 32 semanas ou mais, diminuindo as complicações do parto pré-termo extremo.

\section{ABSTRACT}

Purpose: to analyze the effects of prenatal and perinatal complications and the neurological development of surviving twins when the other had died in utero.

Methods: fourteen cases of twin pregnancies where one of the twins had died during the pregnancy were analyzed. These patients gave birth between 1988 and 1994 and were subsequently followed-up by the Department of Obstetrics, Pathology Division, at the Hospital das Clinicas, Faculty of Medicine of Ribeirão Preto, University of São Paulo. Data from prenatal and perinatal records as well as findings from the deceased twins' autopsies were analyzed. In 1996, requests were made for the children to have a neurological examination as part of the study. The examination included developmental assessment and pathological signs in the motor, sensory and sensitivy areas and superior cortical functions such as praxis and agnosia.

Results: ten of the fourteen contacted subjects complied with the request for neurological examination. Of the ten examined children only one had abnormal neurological findings, presenting a light degree of spastic paresis of the left leg. The pregnancy evaluation showed five cases of monochorionic placenta and one case of monoamnionic pregnancy; six of the fourteen cases reached full-term. In six cases (42.8\%) one of the fetus died during the second trimester and in the other they died during the third trimester. Only one newborn, who had Apgar 0 at the first minute, developed neurological sequelae.

Conclusion: the neurological problem of one fetus may be a consequence of the perinatal complications that this fetus developed. The other newborns did not develop sequelae, possibly because of the conservatory management, trying to make the pregnancy reach 32 weeks or more, thus decreasing the complications of preterm delivery.

KEY WORDS: Twin pregnancy. Fetal death. Neurological sequelae.

\section{Referências}

1. Scheller JM, Nelson KB. Twinning and neurologic morbidity. Am J Dis Child 1992; 146:1110-3.

2. Machado MH, Clode N, Graca LM, Cardoso CG. Hypertension associated with pregnancy. Epidemiologic study of 311 consecutive cases. Acta Med Port 1996; 9:7-14.

3. Kragt H, Huisjes HJ, Touwen BC. Neurological morbidity in newborn twins. Eur J Obstet Gynecol Reprod Biol 1985; 19:75-9.

4. Fusi L, Gordon H. Twin pregnancy complicated by single intrauterine death. Problems and outcome with conservative management. Br J Obstet Gynaecol 1990; 97:511-6.

5. Burguet A, Monnet E, Roth $\mathrm{P}$, et al. Neurodevelopmental outcome of premature infants born at less than 33 weeks of gestational age and not cerebral palsy at the age of 5 years. Arch Pediatr 2000; 7:357-68.

6. Denbow ML, Battin MR, Cowan F, Azzopardi D, Edwards AD, Fisk NM. Neonatal cranial ultrasonographic findings in preterm twins complicated by severe fetofetal transfusion syndrome. Am J Obstet Gynecol 1998 Mar; 178:479-83.

7. Vial Y, Hohlfeld P. Intrauterine death in twin pregnancies. Schweiz Rundsch Med Prax 1999; 88:1435-8. 
8. Hod M, Merlob P, Friedman S, Ovadia J. Single intrauterine fetal death in monoamniotic twins due to cord entanglement. Clin Exp Obstet Gynecol 1988; 15:63-5.

9. Paludetto R. Neonatal complications specific to twin (multiple) births (twins transfusion syndrome, intrauterine death of cotwin). J Perinat Med 1991; 19 Suppl 1:246-51.

10.Schroder W. Intrauterine fetal death - analysis of incidence and causes in an unselected group of 199 twin pregnancies 1985-1989. Geburtshilfe Frauenheilkd 1991; 51:351-4.

11.Haverkamp F, Lex C, Hanisch C, Fahnenstich H, Zerres K. Neurodevelopmental risks in twin-totwin transfusion syndrome: preliminary findings. Eur J Paediatr Neurol 2001; 5:21-7.

12.Axt R, Mink D, Hendrik J, Ertan K, von Blohn M, Schmidt W. Maternal and neonatal outcome of twin pregnancies complicated by single fetal death. J Perinat Med 1999; 27:221-7.

13.van Heteren CF, Nijhuis JG, Semmekrot BA, Mulders LG, van den Berg PP. Risk for surviving twin after fetal death of co-twin in twin-twin transfusion syndrome. Obstet Gynecol 1998; 92:215-9.

14.Yoshida K, Soma H. Outcome of the surviving cotwin of a fetus papyraceus or of a dead fetus. Acta Genet Med Gemellol (Roma) 1986; 35:91-8.
15.Bejar R, Vigliocco G, Gramajo H, et al. Antenatal origin of neurologic damage in newborn infants. II. Multiple gestations. Am J Obstet Gynecol $1990 ; 162: 1230-6$.

16.Fusi L, McParland P, Fisk N, Nicolini U, Wigglesworth J. Acute twin-twin transfusion: a possible mechanism for brain-damaged survivors after intrauterine death of a monochorionic twin. Obstet Gynecol 1991; 78:517-20.

17. Goldberger SB, Rosen DJ, Shulman A, Bahary C, Fejgin MD. Conservative approach to multiple pregnancy with intrauterine fetal death of one or more fetuses. Int $\mathrm{J}$ Gynaecol Obstet 1991; 34:367-72.

18. Gaucherand P, Rudigoz RC, Piacenza JM. Monofetal death in multiple pregnancies: risks for the co-twin, risk factors and obstetrical management. Eur J Obstet Gynecol Repord Biol 1994; 55:111-5.

19.Hanna JH, Hill JM. Single intrauterine fetal demise in multiple gestation. Obstet Gynecol 1984; 63:126-30

20.Feiks A, Scholler J, Rehacek G, Grunberger W. Fetal death in multiple pregnancy: implications for obstetrics and literature review. Z Geburtshilfe Perinatol 1992; 196:44-6.

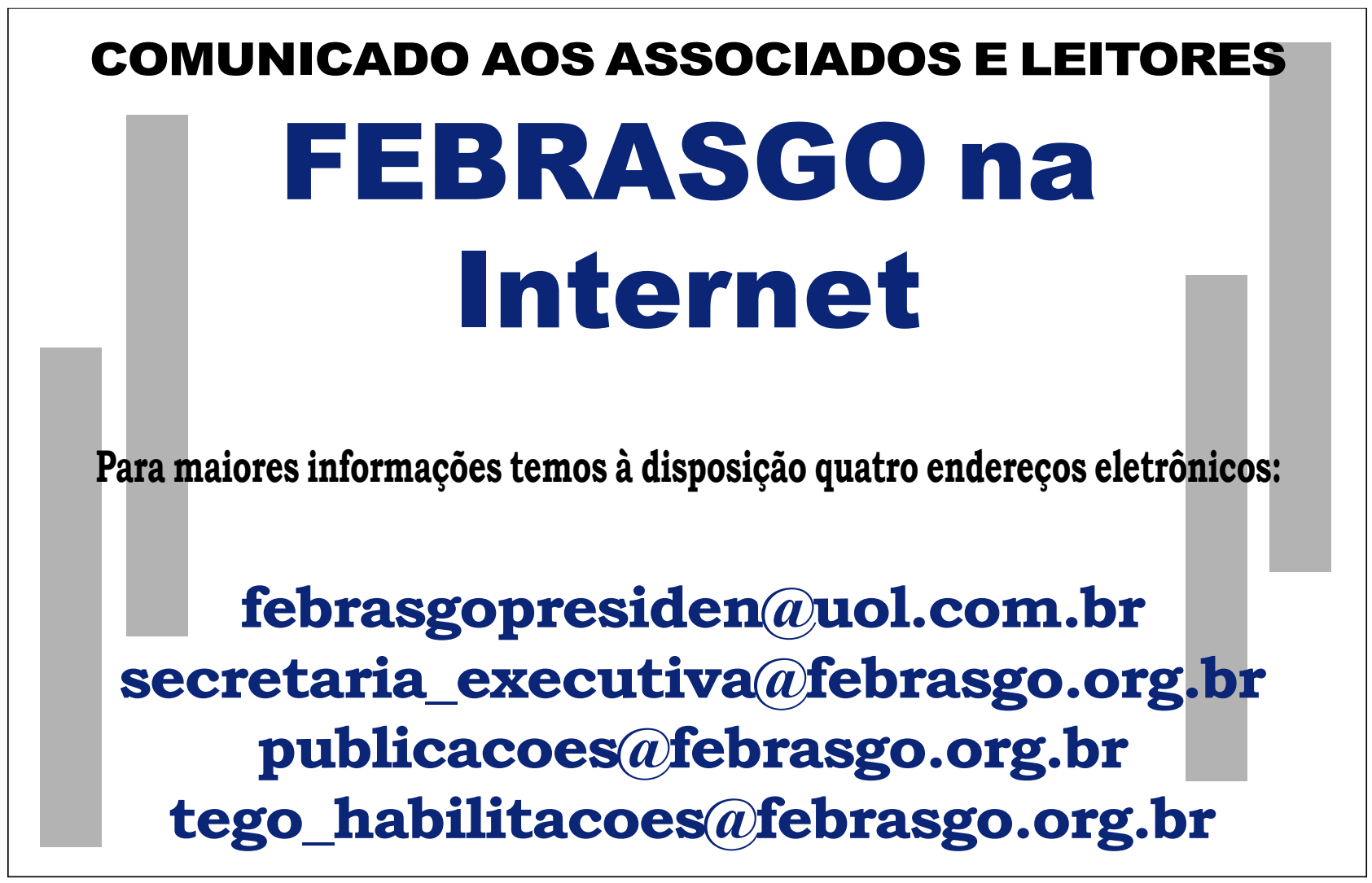

